\title{
Viscosity calculation of polydisperse branching polymers near gel point
}

\author{
D. Sievers \\ Institut für Theoretische Physik, Cologne University, D-5000 Köln 41, West Germany
}

(Reçu le 24 juillet 1980, accepté le 23 septembre 1980)

\begin{abstract}
Résumé. - La viscosité des polymères branchés est calculée à partir de la relation d'interpolation de Marrinan et Hermans dont certaines constantes dépendent à la fois des effets de volume exclu et de la limite de l'écoulement libre. Une étude plus soignée des constantes utilisées montre que le premier choix est mieux adapté au voisinage du point de gelation et conduit soit à une divergence logarithmique soit à une limite finie de la viscosité suivant la théorie utilisée. Les valeurs théoriques de la viscosité intrinsèque ainsi calculées atteignent environ 10 à $20 \mathrm{~cm}^{3} / \mathrm{g}$ pour des distances caractéristiques du point de gel, elles sont en accord avec des valeurs expérimentales de Eschwey, Burchard et Whitney.
\end{abstract}

\begin{abstract}
The viscosity of randomly branched polymers has been calculated based on an interpolation formula by Marrinan and Hermans, which contains dependent on certain coristants both the so called excluded-volumeeffect and the free-draining limit. Closer inspection of the constants used shows that the first choice is better direct near the gel point and yields a logarithmic divergence of the viscosity or a finite limit depending on the gelation

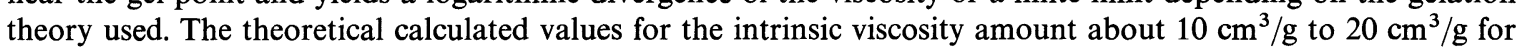
typical distances from the gel point in accordance with some experimental data of Eschwey, Burchard and Whitney.
\end{abstract}

1. Introduction. - Staudinger (1930) already called attention to the utility of viscosity measurements on dilute polymer solutions as a means of characterization. High polymer molecules possess the unique capacity to greatly increase the viscosity of the liquid in which they are dissolved. The viscosity $\eta$ of the solution contains the viscosity of the solvent $\eta_{0}$ and a contribution from the frictional effects of the polymer molecules. The ratio $\left(\eta-\eta_{0}\right) / \eta_{0} . c$ (with $c$ : polymer concentration in $\mathrm{g} / \mathrm{cm}^{3}$ of solution) is a measure of specific capacity of the polymer to increase the relative viscosity $\eta / \eta_{0}$. The limiting value of this ratio at infinite dilution is called the intrinsic viscosity :

$$
[\eta]=\lim _{c \rightarrow 0}\left(\frac{\eta-\eta_{0}}{\eta_{0} \cdot c}\right) .
$$

Kirkwood and Riseman [1] formulated a theory for linear high polymers.

Debye and Bueche [2] developed a molecular model consisting of a sphere with uniform density distribution. More recently percolation theory [3] has been applied to polymer systems. There a periodic lattice is considered, where bonds between nearest neighbouring sites are randomly closed with probability $p$. The groups of connected sites are called clusters. We are concerned here with gelation of randomly branched polymers [4], which is usually treated by a Bethe lattice approximation [4]. At the percolation threshold $p_{c}$ (gel point) an infinite cluster appears, which characterizes the gel phase of polymers. Percolation theory gives values of critical exponents like $\beta, \sigma, \rho, \tau, v$, which obey certain scaling laws summarized e.g. in reference [3].

A detailed composition of methods to treat polymer systems is the new book of de Gennes [5]. Recent discussions of gelation are given by Schmidt and Burchard [6] and Stauffer [7].

2. Calculation. - It has been shown by Marrinan and Hermans [8], that the results of the Debye Bueche theory and the Kirkwood Riseman theory may be rewritten for polydisperse systems in a interpolation formula dependent on two constants $A$ and $a$ :

$$
[\eta]=A\left\langle R^{2}\right\rangle_{\mathrm{w}} \frac{1}{\left(1+a\langle M\rangle_{\mathrm{n}} \frac{\left\langle R^{2}\right\rangle_{\mathrm{w}}}{\left\langle R^{3}\right\rangle_{\mathrm{n}}}\right)}
$$

with the weight average $\langle\ldots\rangle_{w}$ and the number average $\langle\ldots\rangle_{n}$. In polydisperse systems we require averages over the whole distribution of clusters present ; in particular, the number and weight averages are defined as follows : 


$$
\begin{gathered}
\left\langle R^{3}\right\rangle_{\mathrm{n}}=\sum_{s} n_{s} R_{s}^{3} / \sum_{s} n_{s} \\
\left\langle R^{2}\right\rangle_{\mathrm{w}}=\sum_{s} s . n_{s} R_{s}^{2} / \sum_{s} s . n_{s} .
\end{gathered}
$$

Here $n_{s}$ is the number fraction of clusters with $s$ monomers(s-mer) and $R_{s}^{2}$ is the mean square radius of $s$-mer fraction consisting of various structural isomers. The typical radius $R_{s}$ of $s$-clusters varies with size $s$ at the gel point as [9] :

$$
R_{s}=b \cdot s^{\rho} \text {. }
$$

For the fraction of $s$-clusters $n_{s}$ we write [3] :

$$
n_{s}=q_{0} s^{-\tau} f(z) \text { with } z=\left(p-p_{\mathrm{c}}\right) s^{\sigma} .
$$

The function $f(z)$ has to satisfy the following conditions :

$$
\begin{aligned}
f(0) & =1 \\
\lim _{z \rightarrow \pm \infty} f(z) & =0 .
\end{aligned}
$$

We considered two types of $f$-functions [10] :

$$
f_{1}(z)=\left\{\begin{array}{lll}
0 & \text { for } & s>N \\
1 & \text { for } & s \leqslant N
\end{array}\right.
$$

With $f_{1}(z)$ diverging s-sums like $\left(\sum_{s=1}^{\infty} n_{s} \ldots\right)$ are approximated by finite sums $\left(\sum_{s=1}^{N} n_{s} \ldots\right)^{s=1}$, where terms with $s \approx N$ give the main contribution. The critical behaviour of $N$ at the sol gel threshold is described by the exponent $\sigma$ :

$$
N \propto\left|p-p_{\mathrm{c}}\right|^{-1 / \sigma} .
$$

Here $\sigma \approx 1 / 2$ in both classical and percolation theory, whereas $\rho=1 / 4, \tau=5 / 2$ classically, $\rho=0.53, \tau=2.2$ in percolation.
The second f-function, which we considered, is :

$$
f_{2}(z)=1.55 \exp \left(-\frac{\ln 1.55}{(0.834)^{2}}(z+0.834)^{2}\right)
$$

(for equation (8) see also reference [11]).

In the case $1 \ll\langle M\rangle_{\mathrm{n}}\left\langle R^{2}\right\rangle_{\mathrm{w}} /\left\langle R^{3}\right\rangle_{\mathrm{n}}$ we get from equation (1) :

$$
[\eta] \propto \frac{\left\langle R^{3}\right\rangle_{\mathrm{n}}}{\langle M\rangle_{\mathrm{n}}} .
$$

This proportionality was already found by hydrodynamic treatments of Flory [4] and was called the limit of excluded-volume (e.-v.). This case describes a situation, in which the polymer beads form a hydrodynamic sphere impenetrable to the solvent. A calculation of the averages, while $s$-sums are approximated by integrals yields : [ $\eta] \propto \ln N \propto \ln |\varepsilon|$ with percolation exponents in three dimensions (10a) and $[\eta] \propto$ const. with classical exponents $(10 b)$. $\left(|\varepsilon|=\left|p-p_{\mathrm{c}}\right|\right.$ is the distance from the gel point.)

In the case 1$\rangle\langle M\rangle_{\mathrm{n}}\left\langle R^{2}\right\rangle_{\mathrm{w}} /\left\langle R^{3}\right\rangle_{\mathrm{n}}$ however equation (1) leads to :

$$
[\eta] \propto\left\langle R^{2}\right\rangle_{\mathrm{w}} .
$$

This limit is called free-draining (f.-d.) [4].

A f.-d. molecule is a pearl string consisting of beads, which show very small frictional effects during the movement of the polymer molecule relative to the solvent medium [4]. Therefore the solvent streams through the polymer almost unperturbed by it. A calculation of the average $\left\langle R^{2}\right\rangle_{\mathrm{w}}$ yields for the f.-d. limit : $[\eta] \propto \varepsilon^{\beta-2 v}=\varepsilon^{1.3}$ with three-dimensional percolation exponents $(12 a)$ and $[\eta] \propto \ln |\varepsilon|$ with classical exponents $(12 b)$.

In further calculation of equation (1) we obtained for $p \lesssim p_{\mathrm{c}}$ :

$$
[\eta]=A b^{2} q_{0} \beta \delta \cdot \int_{0}^{-\infty} \mathrm{d} z z^{-\beta+2 v-1} f(z) \cdot|\varepsilon|^{\beta-2 v}\left[1-\frac{a m}{b} \int_{0}^{-\infty} \mathrm{d} z z^{-\beta+2 v-1} f(z) \cdot \frac{|\varepsilon|^{\beta-2 v}}{\ln |\varepsilon|}\right]^{-1}
$$

where $m$ is the molecular weight of a monomer.

By using $f_{2}(z)$ the most realistic of the $f$-functions considered and by calculating the constants $A, a$ from data of reference [8] and $b$ from data of [9] we found :

thus

$$
\begin{aligned}
A & =2 \times 10^{14} \mathrm{~cm} / \mathrm{g} \\
a & =6 \times 10^{-11} \mathrm{~cm} \\
b & =5 \times 10^{-8} \mathrm{~cm} \\
q_{0} & =0.2
\end{aligned}
$$

$$
a m / b=0.12 \text { with } m=100 .
$$

(14b) and (14e) are consistent with reference [13]. (14c) means that $b$ is half of the lattice constant of the percolation lattice, which is estimated to $10 \AA$. (14d) refers to [3].

For the intrinsic viscosity we obtained therefore :

$$
\begin{aligned}
{[\eta]=0.56 \mathrm{~cm}^{3} / \mathrm{g} \cdot|\varepsilon|^{-1.3} \times } \\
\times\left[1-3.4 \times 10^{-3} \cdot m \cdot \frac{|\varepsilon|^{-1.3}}{\ln |\varepsilon|}\right]^{-1} .
\end{aligned}
$$

Close to the gel point this result shows that (with monomer molecular weights in the range of $m=100$ ) 
the e.-v. limit should be preferred and gives :

$$
[\eta]=-\frac{1.7 \times 10^{2}}{m} \ln |\varepsilon| \mathrm{cm}^{3} / \mathrm{g} .
$$

De Gennes however gives by hydrodynamic considerations [12] as a description of viscosity near the sol gel transition a proportionality of the intrinsic viscosity to the weight average $\left\langle R^{2}\right\rangle_{\mathrm{w}}$, which is the f.-d. limit equation (11) with a $\varepsilon$-dependence $(12 a)$ in comparison with (16).

The theoretical results may be compared directly with experimental data. Eschwey and Burchard [14] showed for divinyl benzene (DVB) $(m=131)$ with varying amounts of styrene $(m=104)$, that the intrinsic viscosity ranges from about $10 \mathrm{~cm}^{3} / \mathrm{g}$ to $30 \mathrm{~cm}^{3} / \mathrm{g}$ in a molecular weight interval of $10^{6}$ to $10^{7}$. Also Whitney and Burchard found similar results for the copolymers of methyl-methacrylat with its related polyfunctional monomers ethylene dimethacrylat (PPM) in acetone [14]. With $\varepsilon=10^{-3}$ we obtain for $m=117.5$ (average monomer molecular weight of DVB and styrene) from equation (16) the theoretical value :

$$
[\eta]=10 \mathrm{~cm}^{3} / \mathrm{g} .
$$

The described theoretical method still neglects interaction between different macromolecules, which has to be improved. A plausible assumption for viscosity with such interactions was suggested by de Gennes [12] and to some extend confirmed experimentally by Adam et al. [15] and Dumas and Bacri [16]. These possibilities are outside the scope of the present paper.

Furthermore the percolation theory until now dependent on a rigid lattice has to be formulated in a continuum independent of certain lattice structures.

Acknowledgments. - We thank D. Stauffer for suggesting this work, reading the manuscript and many helpful discussions. W. Burchard drew our attention on reference [8].

\section{References}

[1] Kirkwood, J. G. and Riseman, J., J. Chem. Phys. 16 (1948) 565.

[2] Debye, P. and Bueche, A. M., J. Chem. Phys. 16 (1948) 573.

[3] Stauffer, D., Phys. Rep. 54 No 1 (1979).

[4] Flory, P. J., Principles of Polymer Chemistry (Cornell Univ. Press) 1975.

[5] De Gennes, P. G., Scaling Concepts in Polymer Physics (Cornell Univ. Press) 1979.

[6] Schmidt, M. and Burchard, W., submitted to Macromolecules.

[7] Stauffer, D., paper presented at Stat. Phys. 14, to be published in Physica.

[8] Marrinan, H. J. and Hermans, J. J., J. Phys. Chem. 65 (1961) 385.
[9] Peters, H. P., Stauffer, D., Hölters, H. P. and LoeweNICH, K., Z. Phys. B. 34 (1979) 399.

[10] Sievers, D., Staatsexamensarbeit, Cologne University (1980).

[11] Hoshen, J., Stauffer, D., Harrison, R. J., Bishop, G. H. and QuinN, G. D., J. Phys. A 12 (1979) 1285.

[12] De Gennes, P. G., C. R. Hebd. Séan. Acad. Sci. Paris 286B (1978) 131.

[13] Dobson, G. R. and Gordon, M., J. Chem. Phys. 41 (1964) 2389

[14] Eschwey, H. and Burchard, W., Polymer 16 (1975) 180 and J. Polym. Sci., Polym. Symp. 53 (1975) 1.

WhitNeY, R. S. and BURCHARD, W., Makromol. Chem. 181 (1980) 869.

[15] Adam, M., Delsanti, M., OKasha, R. and Hild, G., J. Physique Lett. 40 (1979) L-539.

[16] Dumas, J. and BACRI, J. C., J. Physique Lett. 41 (1979) L-279. 